\title{
Employing Interpretivism in Social Work Research
}

\author{
Venkat Pulla ${ }^{1, *}$, Elizabeth Carter $^{2}$ \\ ${ }^{1}$ Senior Lecturer, Social Work Discipline, Australian Catholic University, Brisbane, Australia \\ ${ }^{2}$ Research Associate at the Brisbane Institute of Strengths Based Practice, Brisbane, Australia
}

\begin{abstract}
Interpretivism is a research paradigm that is associated with qualitative research. A tradition of Interpretivism can be linked to the ethnographies of non-western peoples in the 19th century and continues to be a popular way to explain human behaviour and social phenomenon today. The interpretivist view invites the researcher to investigate meaning behind the understanding of human behaviour, interactions and society. This involves the researcher attempting to develop an in-depth subjective understanding of people's lives. The difference between the natural sciences and social sciences is that the natural sciences requires the researcher to observe and interpret natural processes but the social sciences requires the researcher to observe a phenomenon and understand the interpretations that individuals have already made. Social work research aims to explain human behavior and social phenomenon and not to focus on isolating and objectively measuring variables or on developing generalizations. Social work research requires a focus on gaining an empathic understanding of an individual's feelings and the meaning that they give to everyday life with the goal to gaining greater understanding of the individual's behavior. For this reason, interpretive research is well suited to social work research. In this introductory paper, the authors present the validity of interpretive research and the value of using it in social work research.
\end{abstract}

Keywords Interpretivism, Qualitative Research, Grounded Theory Social Work

\section{Introduction}

In 2014 our world population was over 7 billion people, each with their own individual life stories which are made up in part of their specific life experiences. No two stories will be the same because no two lives are ever lived, and then internalised, in exactly the same way. Reflexivity, positionality, privilege, situated knowledge and perceptions are intrinsically woven into an individual's life story. They coexist with each other; shape, define and refine each other; and, are dependent upon each other for both their existence and development. It is also crucial to recognise that, either as separate entities or as a connected whole, these concepts are fluid. How do we interpret today's modern society? One description could be that it is an array of colours that represents different characteristics of our society such as ethnicity, gender and spirituality etc. Add to it more individualised factors, such as trauma, self-worth, economic security, gender, LGBT, disabilities etc. Interpretation thus is an ongoing process through such variables based on our lived experiences, individualised interpretations and capacity for introspection. Such descriptions can also lead us to the next question. Can there be one truth? Not necessarily. There are several ways of looking at truth and most often this seems to involve our perceptions. Human interpretation is subjective. Thus as researchers if we ought to describe an interpretivist position of all things that we see, observe and write about our interpretations seem to rest on the nature of how we have captured the social reality that we have seen. Clearly there appears no single or all-embracing truth, but rather multiple truths that are woven, grown, offered and paraded as products of human subjectivity (Harrison, 2014).

When designing a research project the researcher must choose a research method which suits the project and will produce the desired outcomes. If, for example the researcher is interested in choosing to interpret a certain human behaviour or wishes to study the interactions or social relationships and build an in-depth understanding of people's lives, a research method that would be suitable in this case would be the Interpretivist research paradigm. Often it is said that subjective information is based on a person's personal opinions, assumptions, interpretations and beliefs, therefore understanding human behaviour in context of their social and physical environments assumes a level of complexity. A more serious interpretation is required. How we interpret and what decisions are made in a situation. The lens through individual views of both self and the other individuals and the world in general are likely to be impacted by variables such as researcher's life 
experiences, and the environment in which the researcher lives. Life experiences and the environment are subject to continual alterations and variations. Thus, it is certain that interpretations vary from individual to individual and seems to suggest or attest that all truth seems to become relative therefore suggesting that we suggest interpretivist approach assists us better in capturing behavioural phenomena.

Using an interpretivist view, the social work researcher is interested in the meaning that individuals give to understanding other humans and the society that they live in. As a social work researcher you are interested in how people make sense of the world, how you understand behaviours of other people, or understand interactions between other people (Sheppard, 2012; Rubin \& Babbie, 2013). For example, a strike or a public demonstration for conservatively minded people can be seen as a disruptive action, undermining economic productivity, having a negative impact on both workers and company owners. For other less conservative people the strike can be seen as good and justified, protecting worker's rights in the face of, possible exploitation, over growing managerial power and insensitivity to labour legitimate demands or growing inequities in wealth. In this instance, we see that the conservative and less conservative individuals have actively constructed their view of actions and events from their own thoughts that are shaped and coloured over time by their experience, their position and their perceptions of social relations in the society. Their views and subsequent actions consecutively will also be based on the objective evidence that they would like to collect to affirm their view. They would be selective in their choice of collecting evidence and selective also in interpreting in line with their original contentions or orientation. Objective evidence, to reiterate constitutes all information based on measurable facts but on researcher's own subjective understanding of human experience. Thus it is evident that we begin working with these subjective meanings that we are able to use in the social world involving ourselves as researchers while acknowledging our own existence. We move on reconstructing them with a view to understand them and possibly avoid distorting them and using them to create theories (Goldkuhl, 2012). Our premise to begin with is all that we have observed and interacted is real or truth. Clearly that the lens in which an individual views both self and others is variable; and that despite our best intentions, the undertaking of our positionality is still fraught with biases and pitfalls (Townson, Pulla, 2015).

\section{A Brief History of Interpretivism}

It is said that the foundations of interpretivism probably began with the works of Max Webber (1864-1920) and Alfred Schutz (1899-1959) who attempted to establish an objective science of the subjective. Their aim was to produce a form of verifiable knowledge of the meanings that make up and illustrate the social world. They focused on the more basic or inherent features, character and qualities of meaningful social action and how a meaning can be attributed. For the purposes of our understanding we will use a simpler defined boundary for social action as being individual or group behaviour or action that involves interaction with other individuals or groups. Webber appeared busy concentrating on generalizing social actions rather than establishing the actual meaning that an individual may offer to social action. This meant that social action could be explained and understood by constructing models of typical meanings used by typical social actors engaged in typical courses of action in typical situations. Webber's work attempted to create causal explanations by linking statistical uniformities to interpretive understanding. For example, if an individual had a good education than they would be able to find a decent job. Schultz on the other hand, recognised the role that social actors (individuals) have in determining social actions (what they do) and argued that these must be taken into consideration when producing an adequate theory. Schultz argued that it is the social actor's not the researcher's point of view that is the basis of any accounts of social life that the researcher is interested in. Interpretivism, was thus born and grew into a research model that is associated with qualitative research and can be linked to the ethnographies of non-western peoples in the $19^{\text {th }}$ century and continues to be a popular way to explain human behaviour and social phenomenon even today (Padgett, 2008). We are aware that the ethnographic tradition was generally passed on orally in that researchers 'learnt by doing' and by the beginning of $20^{\text {th }}$ century ethnographers began producing a rich and varied body of fascinating accounts in qualitative research. Likewise, Grounded theory a result of Glaser and Strauss pioneering efforts further enhanced the quality of ethnographic accounts and systemized the approach to producing ethnographies. There are many qualitative methods that are used today by social work researchers and other social researchers in their studies of social phenomena to gain an understanding of the social worlds that people live in.

\section{Linking Interpretivism and Other Qualitative Methods}

A tradition of interpretivism can be linked to the ethnographies of non-western peoples in the 19th century and continues to be a popular way to explain human behaviour and social phenomenon today. The interpretivist view invites the researcher to investigate meaning behind the understanding of human behaviour, interactions and society. This involves the researcher attempting to develop an in-depth subjective understanding of people's lives. Many researchers are happy with the positivist use of precise quantitative data resulting in rigorous and objective research as the only logical way to collect scientific 
information, which is the process commonly used in the natural sciences. However, the use of this approach in the social sciences is problematic because it is impossible to successfully and clearly empirically measure and interpret the complexity of human behaviour and the interactions between the individual and their social and natural environments. The difference between the natural sciences and social sciences is that the natural sciences requires the researcher to observe and interpret natural processes but the social sciences requires the researcher to observe a phenomenon and understand the interpretations that individuals have already made. For this reason, interpretive research does not focus on isolating and objectively measuring variables or on developing generalizations. Instead it focuses on gaining an empathic understanding of an individual's feelings and the meaning that they give to everyday life with the goal to gaining greater understanding of the individual's behaviour.

Interpretivism sits comfortably in qualitative research particularly with methods such as ethnography and grounded theory, alongside narrative analysis, constructivism, phenomenological approaches that are relatively new to the scene. All qualitative studies seek to understand the individual and their personal interpretations of the subject's experience. The research involves viewing the individual in a holistic manner taking into account the context of the person's experience. For example, the researcher could consider the impact of health, housing, culture, relationships, or environmental issues when investigating the human experience. These are only a few examples and it is up to the researcher to be aware of and explore with the individual subject other issues that may be contributing to an individual's experiences. Qualitative researchers are deeply involved in the data collection and must be sensitive and flexible to follow the thoughts of the person being interviewed to gain an in-depth understanding of the individual's experiences. For instance in Ethnography a description of a culture and understanding another way of life is generated (Krueger \& Neuman, 2006). This involves the researcher attempting and developing an understanding of the society or even social phenomena that is being studied from the inside rather than from an outside perspective. A term that closely describes this process is called immersion. The researchers become part of or immerse themselves in the society that is being studied (Carey, 2012). Some researchers have advocated a way to achieve this by adopting an insider role within the community or by gradually being socialised into the group (O'Reilly, 2009). The fieldwork or data gathering is conducted over a long period where the researcher actively participates within the environment of the research participants (Carey, 2012). This means that the researcher becomes a participant and an observer, improvising as they move through the research process (Harrison, 2014). Moving from what is seen and heard to what is meant is at the centre of ethnography. For example, research on local gangs may involve the researcher joining the gang and participating in the gang's activities while observing and gaining insight from the gang's members. The researcher may also interview other community members that have contact or are affected by the gang. For example, a quantitative study on homeless people may assist with giving information on the numbers that need immediate resources but qualitative research would investigate the individual personal experience of homelessness and the factors that contribute to this social phenomenon.

The researcher can gain insight into the factors that may lead to someone becoming homeless. Qualitative research is clearly good at looking at social processes over a period of time and to gain an understanding of the underlying social interactions that have contributed to experiences. For example, a researcher could examine a riot and after by interviewing individuals, who were affected by the riot, to understand their interpretation of the social interactions that led to the riot.

The Daily Telegraph, a News Paper reported that some Cronulla locals had temporarily abandoned their homes, while others had barricaded themselves indoors, fearful of further revenge attacks and retaliatory rioting in their suburb. They expressed their anger and sorrow about the changes wrought on their suburb and a few revealed they were thinking of moving away altogether. One young woman summed up residents' feelings, saying: "I don't want to live in fear ... I want my freedom back." The Sydney Morning Herald published a feature letter from one local who condemned the "Aussie" rioters' behaviour. Far from protecting the beach, she said "women do not feel safer. We are scared. We are sickened ... Don't defend our way of life by preaching hatred, violence and racial vilification ..." Local surfer girls said it was the behaviour of young men on the beach, not their race or religion that was an issue for them. They felt the "protective" response of the "Aussies" was just an excuse to get drunk and aggro. Surf lifesaving events at Cronulla beaches were cancelled after clubs expressed their concerns about sending members into potential conflicts. Cronulla lifesavers revealed that dealing with aggression and harassment from beachgoers was a normal part of their experience, but it had "been getting worse the past few months" (Reportingdiversityorg.au, 2016)

Thus the qualitative process is not only a data collecting activity but a theory generating activity. The researcher will not generally start with a defined hypothesis to be tested. The project will generally start with a question or topic to be researched and as the researcher proceeds with observations and interviews data silts through as if we used a sieve. The ongoing process of an inbuilt review of the data reveals more information that generates questions to be answered. Our research, as a result of intensive 
interviews' invites the interviewer to revisit experience(s) of people particularly what might possibly be present underneath the surface ; undertake a re-exploration of the statement or topic or even return to an earlier point. Charmaz, in her grounded theory discourse refers to this act as a validation process of participant's humanity, perspective or action (Charmaz, 2009: 26) and this is indeed a very powerful process.

\section{Carrying Interpretivism through Ethnography}

The ethnography research process is not just a description of what the researcher sees each day but capturing an understanding of the influence of and engagement with the wider social and cultural forces. Examples include the impact of policies enacted by organisations on people, subjective and cultural priorities that people have that impact on their daily roles, unseen informal processes embedded in language, relationships or status, or different ways that individuals express their identities or emotional intelligence (Carey, 2012). It is important that the culture being researched is understood on their own terms and not judged by the researcher's values and beliefs (Padgett, 2008). To address this during the research process the researcher should reflect on the relationship between the researcher and the researched and acknowledge the researchers own subjective interpretation of the events and interactions being observed (Carey, 2012).

The first step in ethnographic research is to create a brief statement which expresses what the research project hopes to explore and address. This statement is important because even though it may be revised or adapted once in the field it helps to focus the research activities and plans. The researcher then needs to decide on where the research will take place and what communities will give the information that will address the project aims. It is then important to decide what, where and when data will be collected. Ethnographic notes should be clear and concise about what has been observed. The researcher will also use diary notes to analyse information and the researcher's reflections. It is also important to consider access to the intended population as the researcher may have permission to do the study by authorities but individual's within the community may be suspicious and uncooperative which will require the researcher to use communication skills to build relationships. As with all qualitative research the analysis of data is a continuous process throughout the research project and continues until no new information is uncovered.

Harrison (2014) poses some interesting questions that the researcher should reflect on when considering ethnographic research. What does it mean to study someone else's life and what gives the researcher the right to do research on another community? More importantly what is the goal of the research? Is it working in service of the community or other interests? Reflecting on these questions is important when considering ethnographic research as it is important to consider the safety the researcher, for example the risk of embedding into a street gang. On the other hand, consideration should also be given to the dangers that the researcher brings into the community. For example, a researcher may disrupt the status quo of a community causing disruption and in some cases violence (Madison, 2015).

Another method that allows us to utilise interpretivism is also the method of grounded theory. As a qualitative research method it seeks to develop theory that is grounded in the data that is systematically gathered and analysed. During the data collection it is important that there is a continuous interplay between data collection and analysis. Data analysis is non-linear and revolves in a circular process that involves the researcher reanalysis and reinterpretation of data to ensure a valid end product (Pulla, 2014). As the data analysis reveals new patterns or observations the researcher then seeks out more data to compare those observations against the concepts and hypothesis from earlier observations (Rubin \& Babbie, 2014). Researchers that use the Grounded theory approach should make sure that they do not have any preconceived theories in mind before starting on their research project. It is important in this approach that the researcher allows the theories and concepts to emerge from the data. In other words, the research does not begin with a theory; the theory is the outcome (Pulla, 2014). It is for this reason that generally the literature review does not take place early in the research project but continues throughout (Cary, 2012). Interviews are transcribed verbatim and the analysis does not make assumptions but allows the data to narrate the story. This ensures that the data that is collected gives the researcher a clear understanding and interpretation of the interviewees' own perspective (Pulla, 2014).

\section{Qualitative (Interpretivism) vs Quantitative (Positivism)}

The usefulness of qualitative research has been questioned as there are concerns about the generalisability of the research results to other groups (Voyer \& Trondman, 2015). Qualitative research is meant to study a specific issue or phenomenon in relation to a certain population, location or context. It is therefore considered that generalisability of qualitative research is not possible but trends in qualitative research through meta-analysis have been able to increase the possibility of generalisability (Lawrence, 2015). Meta-analysis is the systematic analysis of several qualitative research projects that are examining the same phenomenon. Despite the increase in meta-analysis, the view of many researchers is that the 
positivist use of precise quantitative data resulting in rigorous and objective research is the only logical way to collect scientific information, which is the process that commonly used in the natural sciences (Kreuger \& Neuman, 2006).

The use of a quantitative research approach in the social sciences is problematic because it is impossible to successfully and clearly empirically measure and interpret the complexity of human behaviour and an individual's interactions between their social and natural environments. The use of Quantitative research methods in the natural sciences allows the researcher to observe, measure and interpret natural processes. Research in the social sciences involves observing a phenomenon and understanding the interpretations that individuals have already made (Blaikie, 2004). For this reason, interpretive research does not focus on isolating and objectively measuring variables or on developing generalizations. Instead it focuses on gaining an empathic understanding of an individual's feelings and the meaning that they give to everyday life with the goal to gaining greater understanding of the individual's behaviour (Rubin \& Babbie, 2013). To summarise this quantitative research deals primarily with objective numerical data to test a theory. Qualitative research seeks to answer questions of "how, where, when, who and why" to build a theory by collecting non numerical information and human subjectivity of a phenomenon. In Quantitative research human emotions and perspectives are seen as undesirable biases but in Qualitative research these elements are seen as essential to the research process (Lawrence, 2015).

Interpretive research reveals the views that emerge from the research rather than giving a definite answer (Willis, 2013). Concerns of bias or limitations in the research process can be addressed by the skill of the researcher to be reflexive in and reflect on the research process and how they personally impact the data produced (Voyer \& Trodman, 2015). Reflexivity involves the researcher being self-consciously aware of their impact on the research and research process. In the research setting the researcher is a participant in the interaction between themselves and participants and thus integrally contributes to the data. This involves not only reflection on the interview process but the impact that the researcher has on the whole research process. For this reason, it is important that the researcher reflects on and can clearly explain decisions made and processes used (Willis, 2013).

\section{Conclusions}

Social work needs a strong and robust understanding of human behavior, interactions and society as a foundation for its practice frameworks. This paper looked at the two of the founding qualitative approaches of ethnography, where the researcher studies the phenomenon from the inside rather than the outside, and grounded theory, where the researcher develops theory from systematically collected data. These qualitative approaches assist the researcher to interpret the complexity of human behavior and deliver on finding meaning behind an individual's actions rather than a generalization provided by qualitative research.

If you subscribe to the interpretivist view that social realities are made up of multiple truths depending on the individual's subjectivity, then qualitative research is the perfect way to develop theories on human behaviour. The qualitative research process allows the researcher to systematically collect data through observation, participation and participants' subjectivity and has the ability to address the complexities of understanding human behaviour in context of their social and physical environments. As with any process it is important to also remember that even though both approaches have considerable merit it is important to thoroughly understand limitations and risks involved in using either research method.

Qualitative research has an important role to play in social work practice as the theories generated by this type of research can underpin quality practice that is required by the social work practitioner.

\section{REFERENCES}

Blaikie, N. (2004) Interpretivism. In M. Lewis-Beck, A. Bryman \& T. Liao (edt.) The SAGE Encyclopedia of Social Science Research Methods. Thousand Oaks: SAGE Publications. DOI: http://dx.doi.org/10.4135/9781412950589.n442

Carey, M. (2012). Qualitative research skills for social work: Theory and practice. Surrey, England: Ashgate Publishing Limited. Ebook

Charmaz, K. Constructing Grounded Theory: A Practical Guide through Qualitative Analysis. Sage Publications, Thousand Oaks, CA, 2006.

Goldkuhl, G. (2012). Pragmatism vs interpretivism in qualitative information systems research. European Journal of Information Systems 21, 135-146

Harrison, A. (2014). Ethnography. In P. Leavy (edt.) The Oxford handbook of qualitative research. Oxford Handbooks Online, DOI: 10.1093/oxfordhb/9780199811755.013.011

Kreuger, L. \& Neuman, W. (2006) Social work research methods: qualitative and quantitative approaches: with research navigator. Boston: Pearson/Allyn and Bacon

Lawrence, L. (2015). Validity, reliability, and generalizability in qualitative research. Journal of Family Medicine and Primary Care, 4(3), 324.

Madison, D (2015). Dangerous ethnography. In N. Denzin \& M. Giardina (edt), Qualitative inquiry - Past, present, and future. Walnut Creek: Left Coast Press. Retrieved from E-book library. 
O'Reilly, K. (2009). Key concepts in ethnography. Los Angeles: SAGE. Retrieved from CREDOreference Academic Core.

Oktay, J. (2012). Grounded theory. New York: University Press, Retrieved from Oxford Scholarship Online, DOI: 10.1093/acprof:oso/9780199753697.001.0001

Padgett, D. (2008). Qualitative methods in social work research ( $2^{\text {nd }}$ ed.). Los Angeles, Calif.: Sage Publications

Pulla, V. (2014). Grounded theory approach in social research. Space and Culture, India 2(3), 14-23.

Pulla V. (2016). An Introduction to the Grounded Theory Approach in Social Research. International Journal of Social Work and Human Services Practice, 4, 75 - 81. doi: 10.13189/ijrh.2016.040401.

Reportingdiversity.org.au. (2016). The Cronulla riots - the sequence of events. Journalism in Multicultural Australia: Case studies. Retrieved 10 August, 2016, from http://www.reportingdiversity.org.au/cs_four.pdf

Rubin, A. \& Babbie, E. (2013). Essential research methods for social work ( $3^{\text {rd }}$ ed.), Belmont, CA: Brooks/Cole Cengage Learning

Rubin, A. \& Babbie, E. (2014) Research methods for social work ( $8^{\text {th }}$ ed.). Belmont, California: Brooks/Cole Cengage Learning

Sheppard, M. (2012). Social Work and Social Exclusion. Abingdon, GB: Ashgate. Retrieved from http://www.ebrary.com

Townson, L \& Pulla V, (2015)._Preparing social workers for empowerment: the place of positionality and its continual intricacies. In Venkat Pulla and Bharath Bhushan Mamidi (eds), Some Aspects of Community Empowerment and Resilience, New Delhi, Allied Publishers Pvt. Ltd, 2015, 258 pp., ISBN 978-81-8424-962-0

Voyer, A. \& Trondman, M. (2015). Between theory and social reality: Ethnography and interpretation and social knowledge. Ethnography 0(00), 1-7. DOI: 10.1177/1466138115592415

Willis, K. (2013). Analysing qualitative data. In M Walter (edt), Social research methods ( $3^{\text {rd }}$ ed.), South Melbourne, Victoria: Oxford university press. 